Received: 2016.11 .17

Accepted: 2016.12 .04

Published: 2017.08.23

Authors' Contribution:
A Study Design
B Data Collection
C Statistical Analysis
D Data Interpretation
E Manuscript Preparation
F Literature Search
G Funds Collection

\title{
Clinical Audit of Paediatric Magnetic Resonance Imaging Under Sedation at a Nigerian Tertiary Institution
}

\author{
Afolabi Muyiwa Owojuyigbe ${ }^{1 \mathrm{1AF}}$, Adeleye Dorcas Omisore ${ }^{2 \mathrm{~B} 6 \mathrm{BD}}$, \\ Anthony Taiwo Adenekan 1四, Olusola Comfort Famurewa2四, \\ Oluwagbemiga Oluwole Ayoola ${ }^{2 \text { ॠ }}$, Fredrick Andrew Attah ${ }^{2 \text { बi }}$, \\ Victor Adebayo Adetiloye ${ }^{\text {¿बi }}$
}

${ }^{1}$ Department of Anaesthesia, Obafemi Awolowo University/Obafemi Awolowo University Teaching Hospitals Complex, Ile Ife, Osun State, Nigeria

${ }^{2}$ Department of Radiology, Obafemi Awolowo University, Teaching Hospitals Complex, Ile Ife, Osun State, Nigeria

Author's address: Adeleye Dorcas Omisore, Department of Radiology, PMB 5538, OAUTHC, Ile Ife, Nigeria, e-mail: omisoreadeleye@yahoo.com

\section{Summary}

Background: $\quad$ Magnetic resonance imaging (MRI) in paediatric patients requires them to be calm during the procedure to avoid motion artefacts in the acquired images. Sedation and/or anaesthesia is a way to achieve this. We evaluated all paediatric MRI sedations since installation of an MRI device in our hospital.

Material/Methods: $\quad$ We retrospectively reviewed 69 paediatric MRI sedations performed over a 5-year period using records of patients' biodata, MRI date, indication, findings and scan time, sources of referral, body region scanned, type, dose, related adverse events and route of administration of sedatives as well as image quality.

Results: $\quad$ The median age and weight of the patients were 24 months \{range of 0.3 months (10 days) to 132 months (11 years) $\}$ and $11.5 \mathrm{~kg}$ (range of $2.6 \mathrm{~kg}$ to $42 \mathrm{~kg}$ ), respectively. Males constituted $50.7 \%$ of the patients. Most participants (94.2\%) were in-patients of the hospital, mainly $(60.0 \%)$ referred from the paediatric unit, with slightly over one third (36.2\%) of the studies performed in 2015. The commonest indication and scanned body region were macrocephaly $(18.8 \%)$ and the brain $(76.8 \%)$, respectively. Hydrocephalus (17.4\%) was the commonest MRI finding. Sedation was planned in 66 (95.7\%) patients and was successful in 68 (98.6\%). Midazolam and the IV route were the commonest sedative agent and route of administration, respectively. Image quality determined by age was fair to good in $68(98.6 \%)$ patients with only 1 patient requiring re-scanning due to motion blur. No adverse events with sedation were recorded.

Conclusions: Midazolam via the IV route with or without oral route is the drug of choice for MRI sedation in children in our institution with a success rate of about $99 \%$.

MeSH Keywords: $\quad$ Conscious Sedation • Hospitals, Pediatric • Nigeria

PDF file: $\quad$ http://www.polradiol.com/abstract/index/idArt/902419

\section{Background}

In Nigeria, the awareness of and competence with regard to proper use of magnetic resonance imaging (MRI) appears to be high [1]. MRI has also become the imaging modality of choice for many paediatric conditions, because it is non-invasive, does not use ionizing radiation and provides multi-planar, high-contrast images [2]. However, it requires patients to be completely calm and still during the procedure to avoid motion artefacts in the acquired images [1]. Inability of children between ages of 1 to 5 years to understand and comply with instructions, the problem of physical conditions of patients that result in pain and patients' fearful reactions to loud noise emerging from MRI devices 
and claustrophobia can lead to involuntary and voluntary movements during MRI procedure, which eventually leads to motion artefacts in the acquired images [3]. Therefore, children who undergo MRI scanning frequently need some level of calmness, sleep or analgesia in order to remain still for the procedure. This is usually achieved by sedation (minimal, moderate or deep) and/or general anaesthesia [4].

There are MRI compatible anaesthetic machines and monitors used to provide sedation and monitor patients during MRI procedures. They are, however, expensive and are not available in many MRI centres, especially in low resource setting like ours [4]. Sedation of paediatric patients in such settings can thus be quite challenging.

The ideal sedation protocol is one that has an easy route of administration, with little or no adverse reactions, and allows for a quick and complete recovery [4]. However, there could be a number of potential problems including organizational difficulties in ensuring that the child is admitted, sedated and scanned, all in a timely, coordinated and safe manner.

Sedation is usually required in patients who are aged 5 years or younger [4]. Occasionally, older children who are mentally retarded or in an irritated state may require sedation [4]. It is our departmental policy for children younger than 6 years to be sedated before they come to the MRI suite in our hospital.

In our hospital, we have an institutional sedation protocol for MRI in children. The sedation regimen for each individual patient is determined and administered by the anaesthesiologist based on the drawn protocol and after a consult has been sent by the radiologist.

The aim of this audit was to evaluate all paediatric MRI sedations performed since the installation of an MRI device in our hospital.

\section{Material and Methods}

All cases of paediatric MRI examinations performed under sedation in our facility between January 2011 and December 2015 with an open, 0.2 Tesla, GE MRI scanner were included in this audit.

For this audit review, the following data were collected on standard data sheets: patient's age, gender, weight, date of MRI examination, sources of referral, indication for study, body region scanned, sedative agent used, route of administration, dose of the sedative agent, MRI findings, image quality, total MRI scan time and adverse events during procedure. It was also noted if the sedation was a planned or an emergency one, and if it was successful or unsuccessful.

An established institutional sedation protocol is available in our centre and sedation is performed by the paediatric anaesthesiologist, after a consult has been sent by the radiologist. An intravenous (IV) catheter is inserted so that IV contrast medium or further IV sedating agents may be given, if required.

\section{Institutional sedation protocol}

An institutional sedation guideline for children is in place in our institution. Paediatric anaesthesiologists administered all sedatives and monitored the children throughout the procedure. All sedations were performed in a predesigned area outside the MR suite. Pre-sedation instructions included fasting for at least 6 hours with respect to solid food or meals and at least 2 hours regarding clear non-carbonated fluids. All patients were reviewed by the anaesthesiologist before their MRI procedure. Midazolam was the sedative of choice. A bolus dose of $0.1 \mathrm{mg} / \mathrm{kg}$ IV was used in the majority of patients. Children who we anticipated to be restless during IV cannulation were given the IV formulation of midazolam as an oral preparation at a dose of $0.1 \mathrm{mg} / \mathrm{kg}$ midazolam added to $20 \mathrm{mls}$ of Ribena ${ }^{\circledR}$ (a blackcurrant flavoured un-carbonated drink, which was used as sweetener). Depth of sedation was assessed every 5 minutes using the Ramsay's sedation scale (RSS) [5]. RSS of 3 or 4 was targeted. Non-invasive blood pressure (NIBP) and arterial oxygen saturation ( $\mathrm{SaO} 2)$ of all patients were monitored. Supplemental oxygen was given to all patients. After the first bolus dose of $0.1 \mathrm{mg} / \mathrm{kg} I V / o r a l$, the patients were given a repeat bolus if the RSS of 3 was not achieved after 10 minutes (longer to achieve with oral bolus). Once an adequate sedation level was achieved, the patients were transferred into the MRI suite. If patent woke up during the procedure, the scanning was interrupted to give a repeat bolus. Additional monitoring was done by using an abdominal belt which displayed the respiratory rate on the MRI screen; also a direct observation of the respiratory pattern of the patients through the transparent glass partition was made. Patients were transferred to the recovery area after the MRI procedure and vital signs were monitored at 5 minute intervals until discharge criteria (Aldrete score of 9) [6] were met.

Some paediatric patients who already had paraldehyde in the course of their management for seizure disorders shortly before coming for MRI were not sedated but only monitored during the procedure.

For out-patients, contact address of the referring doctor is usually given to report any observed side effects of sedation, which was explained to the parents or guardians. For in-patients, if complications of sedation occurred, the anaesthesiologist was alerted immediately.

\section{Statistical analysis}

The patients' characteristics (age, gender, weight), source of referral, doses of sedatives used, indications for study, body region scanned were analysed using descriptive statistics and are presented as percentages or medians/ranges where appropriate. The association between image quality, patient age and route of administration of sedatives was analysed using Fischer's exact test; $p \leq 0.05$ was considered statistically significant. 


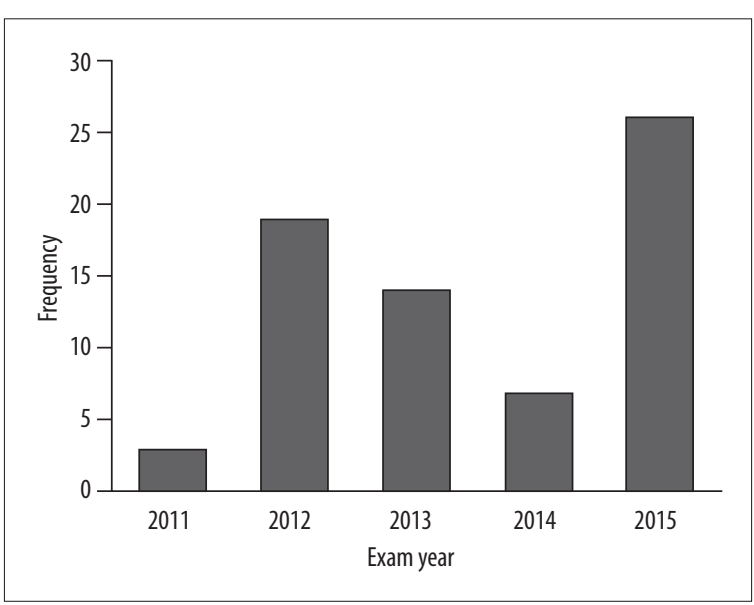

Figure 1. Bar chart showing distribution of paediatric MRls performed under sedation over the 5 -year period

Table 1. Characteristics of the sedated paediatric MRI patients.

\begin{tabular}{lcc}
\hline \multicolumn{1}{c}{ Variable } & \multicolumn{2}{c}{ Outcome } \\
\hline Age: median (range) & $24(0.3-132)$ months \\
\hline Gender: $n$ (\%) & & \\
\hline Male & 35 & $(50.7 \%)$ \\
\hline Female & 34 & $(49.3 \%)$ \\
\hline Weight: median (range) & $11.5(2.60-42.0) \mathrm{Kg}$ \\
\hline Referral: $n$ (\%) & & \\
\hline In patients & 65 & $(94.2 \%)$ \\
\hline Out patients & 4 & $(5.8 \%)$ \\
\hline
\end{tabular}

\section{Results}

\section{Profile of children sedated for MRI}

A total of 69 paediatric MRI cases were performed under sedation over the 5-year period. Slightly over one third (37.7\%) of the MRIs under sedation were done in 2015, while only $3(4.3 \%)$ were done in 2011 which was the year with the lowest number of cases (Figure 1). The average number of cases per year was 13.8.

The median age of the patients was 24 months with a range of 0.3 months (10 days) to 132 months (11 years). Thirtyfive $(50.7 \%)$ patients were males, while $34(49.3 \%)$ were females. The weight of the patients ranged from $2.6 \mathrm{~kg}$ to $42 \mathrm{~kg}$, with a median weight of $11.5 \mathrm{~kg}$ (Table 1 ).

Sixty-five (94.2\%) of the cases were in-patients of the hospital (Table 1) and about $60.0 \%$ of in-patients were referred from the paediatric unit followed by $21.5 \%$ of the inpatients referred from the neurosurgery unit (Figure 2).

\section{Clinical indication/body region scanned/MRI findings}

In this audit, the commonest indication for MRI was macrocephaly, seen in $13(18.8 \%)$ of the patients, and the

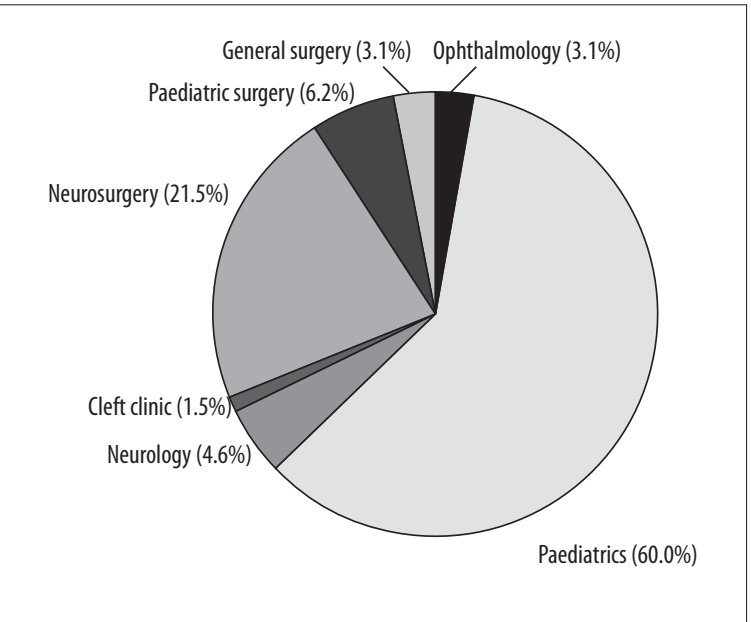

Figure 2. Pie chart showing distribution of the sources of referral of in-patient paediatric MRI examinations performed under sedation.

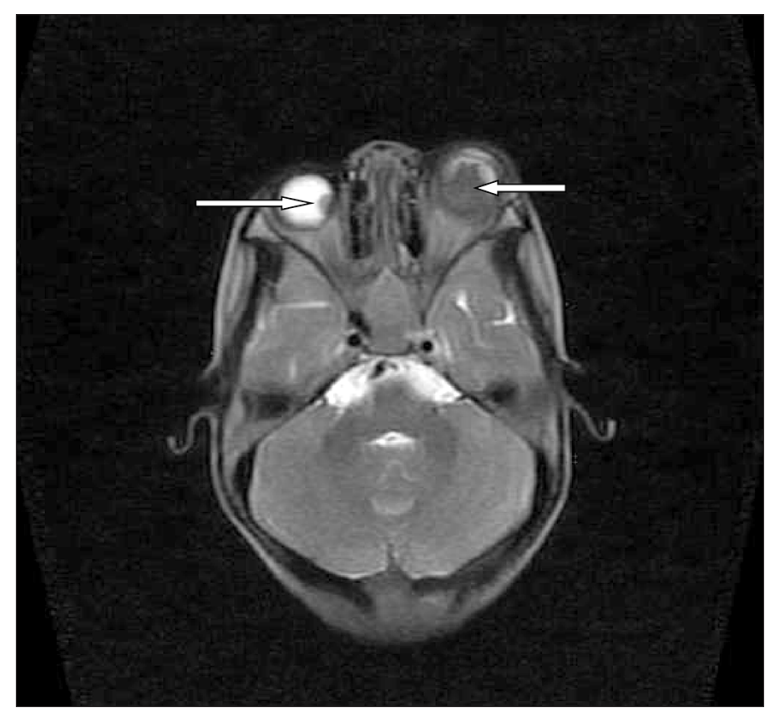

Figure 3. Axial, unenhanced, T2 MRI of the head with good image quality showing a bilateral intraocular, hypointense mass in a 4-year-old female with bilateral retinoblastoma (white arrows).

brain (Figure 3) was the commonest body region scanned, i.e. in $53(76.8 \%)$ of the paediatric patients (Figure 4). Of the remainder, 8 patients (11.6\%) had MRI examinations of the abdomen, 4 (5.8\%) examinations of the spine (1 lumbosacral, 1 lumbar and 2 thoracolumbar), and 4 (5.8\%) an examination of the cranioorbital area (Figure 4).

Hydrocephalus (Figure 5), seen in 12 (17.4\%) patients, was the commonest finding on MRI.

\section{Type/dose/route of administration of sedatives}

Intravenous route alone was used in $54(78.3 \%)$ patients, while $15(21.7 \%)$ patients required both intravenous and oral routes. Midazolam was used for sedation in 66 (95.7\%) patients, while the remaining 3 (4.3\%) patients had paraldehyde which was administered on the wards by the paediatrician (Table 2). The indication for MRI in these 3 patients 


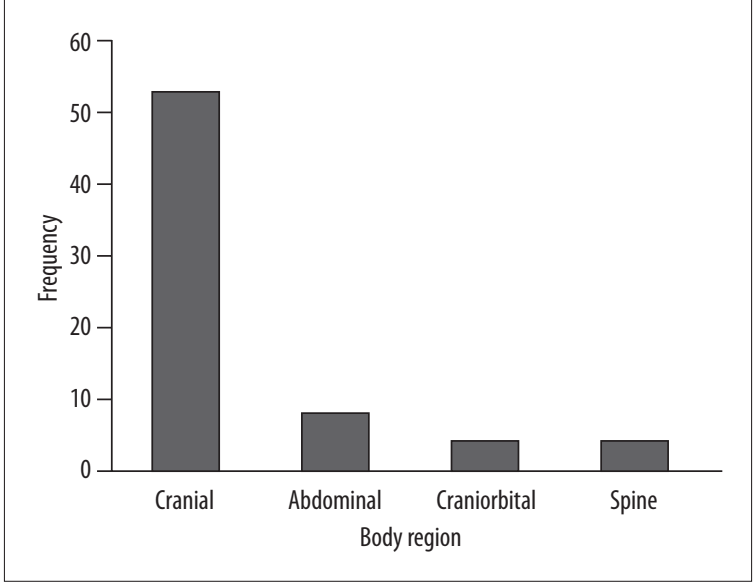

Figure 4. Bar chart showing the frequency of the body region scanned.

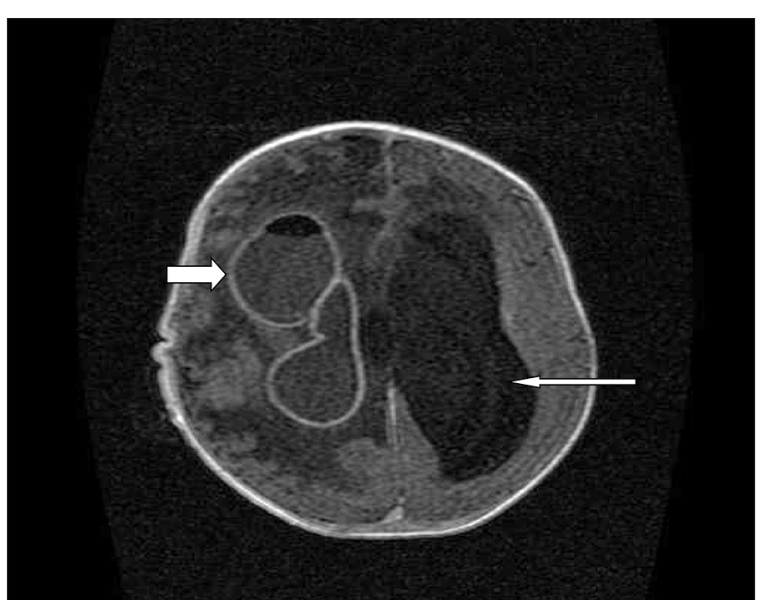

Figure 5. Axial, contrast-enhanced, T1-weighted MRI of the head demonstrating hydrocephalus (long white arrow) with rim enhancement of the air-containing right lateral ventricle (short white arrow) and extensive right cerebral oedema in a 4-month-old male with meningitis complicated by an intraventricular abscess.

was seizure disorder. The doses of midazolam used ranged from 0.6 to $8.4 \mathrm{mg}$.

\section{Outcome of sedation/duration of MRI procedure/image quality/ adverse event}

Sedation was planned in 66 (95.7\%) patients, while 3 patients (4.3\%) had emergency sedation administered. Sedation was successful in about $99 \%$ of patients (Table 2). The median duration of the MRI examinations was 29.5 minutes with a range of 20 minutes to 70 minutes. The quality of MRI images was good (Figure 3) in 61 (88.5\%) and fair in $7(10.1 \%)$ patients. Only $1(1.4 \%)$ patient required rescanning due to poor image quality (Figure 6) from motion blur (Table 2); this examination studied the abdominopelvic region (Figure 6, Table 2).

No patient had an adverse event during the MRI procedure performed under sedation.
Table 2. Characteristics of sedatives used and Image quality.

\begin{tabular}{|c|c|c|}
\hline Variable & \multicolumn{2}{|c|}{ Outcome } \\
\hline Midazolam & 66 & (95.7\%) \\
\hline Paraldehyde & 3 & $(4.3 \%)$ \\
\hline \multicolumn{3}{|l|}{ Route: n (\%) } \\
\hline IV only & 54 & $(78.3 \%)$ \\
\hline IV + Oral & 15 & $(21.7 \%)$ \\
\hline \multicolumn{3}{|l|}{ Dose: median (range) } \\
\hline Midazolam & \multicolumn{2}{|c|}{$3.3 \pm 2.5 \mathrm{mg}(0.6-8.4 \mathrm{mg}$} \\
\hline Paraldehyde & \multicolumn{2}{|r|}{-} \\
\hline \multicolumn{3}{|l|}{ Sedation: $\mathrm{n}(\%)$} \\
\hline Planned & 66 & (95.7\%) \\
\hline Emergency & 3 & $(4.3 \%)$ \\
\hline \multicolumn{3}{|l|}{ Image quality } \\
\hline Good & 61 & $(88.5 \%)$ \\
\hline Fair & 7 & $(10.1 \%)$ \\
\hline Poor & 1 & $(1.4 \%)$ \\
\hline \multicolumn{3}{|l|}{ Repeat study } \\
\hline Yes & 1 & $(1.4 \%)$ \\
\hline No & 68 & $(98.6 \%)$ \\
\hline
\end{tabular}

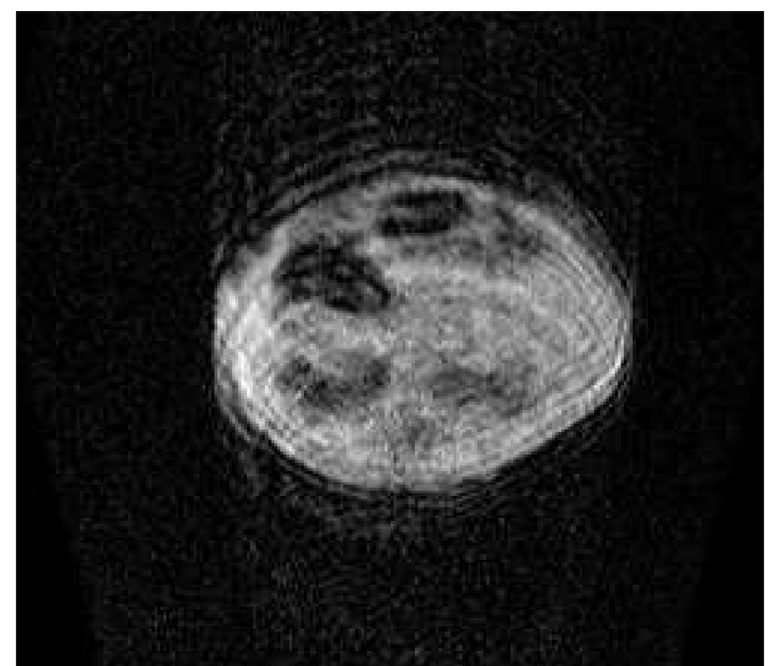

Figure 6. Axial, unenhanced, T1-weighted abdominopelvic MRI with poor image quality resulting from motion blur in a 2-month-old male with a pelvic primitive neuroectodermal tumour (PNET).

The Fischer's exact test showed that image quality was determined by age $(p \leq 0.05)$ of the patients but not by the route of administration $(p=1.00)$, (Table 3 ). 
Table 3. Association between image quality, age of patient and route of administering sedative agent.

\begin{tabular}{|c|c|c|c|c|}
\hline Variable & & lood & Fair/poor & $p$ Value \\
\hline \multicolumn{5}{|l|}{ Patient age* } \\
\hline$\leq 36$ months & & (95.5\%) & $2 \quad(4.5 \%)$ & \multirow{2}{*}{0.023} \\
\hline$>36$ months & 19 & (76.0\%) & $6 \quad(24.0 \%)$ & \\
\hline \multicolumn{5}{|l|}{ Sedation route* } \\
\hline IV only & & (88.9\%) & $6 \quad(11.1 \%)$ & \multirow{2}{*}{1.00} \\
\hline IV + Oral & & $(86.7 \%)$ & $2 \quad(13.3 \%)$ & \\
\hline
\end{tabular}

* Fischer exact test used. Significant $p \leq 0.05$.

\section{Discussion}

Although MRI was introduced into clinical practice in the early 1970s, it only became available in Nigerian Teaching Hospitals in the end of 2005 through a governmental refurbishment in 14 Nigerian Teaching Hospitals.

Our audit shows that on average 13.8 sedated paediatric scans were done yearly in our facility over the 5 -year period of this audit. This amounts to a monthly average of 1.2 paediatric MRI scans done under sedation. The small number of examinations in our audit may be related to the high cost of MRI accompanied by a low economic status of the majority of people in our area. A downtime period of the MRI machine due to industrial strike actions and machine breakdowns may also have contributed to the small number of sedated paediatric MRIs in our facility. Our audit revealed a total of 499 MRI scans performed over 5 years, of which 109 were paediatric scans. This figure is lower than the 832 examinations reported by an audit from a facility with a low-field strength MRI by Ogbole et al. [1] that was performed in another teaching hospital in Southwestern Nigeria over a similar period with an average number of annual scans of 160. This difference may be due to the larger population density and urbanized nature of their host community, as compared to ours. The difference in the audit periods between the Ogbole et al. [1] study (2006-2011) and ours (2011-2016) may also be contributory, however, no recent audits from other Nigerian Teaching Hospitals are available for comparison with our findings.

The age range of patients from our audit is similar to that from the audit carried out by $\mathrm{Chu}$ et al. [4] (10 days to 11 years versus 13 days to 13 years, respectively). Our median age was 2 years while the mean age in the audit by Chu et al. [4] was 3.2 years. In the study by Mazhar and coworkers [7], 60\% of patients were aged between 1 and 5 years. The age of patients influenced image quality in our audit, as patients younger than 36 months had relatively better images than those aged over 36 months. It can be deduced from our audit that younger children probably had less anxiety and were more successfully sedated than their older counterparts, which in turn produced better MRI images.

As expected, the majority (56.5\%) of paediatric patients referred for sedated scans were from paediatric units, suggesting a good utilization of MRI by paediatricians. The commonest indication in our audit was macrocephaly, which is different from the commonest indication from the audit performed in New York by Mazhar et al. [7] - i.e. seizures. Hydrocephalus (Figure 5), which was our commonest MRI finding, was not unexpected as the commonest indication for MRI was macrocephaly in our patients. In the audit by Mazhar et al. [7], incidental findings were most common..

Two sedative agents recorded in our audit were (1) $0.1 \mathrm{mg} /$ $\mathrm{kg}$ oral and/or IV midazolam which was administered by the anaesthesiologist, and (2) IM paraldehyde which was administered by the paediatrician before patients presented in the MRI suite. These 2 drugs were not given in combination. Mazhar et al. [7] used 50-100 mg/kg of oral chloral hydrate and $0.1 \mathrm{mg} / \mathrm{kg}$ of IV midazolam with the majority of patients receiving both of these sedative agents, while Chu et al. [4] used 50-100mg/kg of oral chloral hydrate, IM paraldehyde and $0.1 \mathrm{mg} / \mathrm{kg}$ of IV midazolam. In the audit by Malviya et al. [8], oral chloral hydrate, benzodiazepines, diphenhydramine, meperidine and midazolam were used in $90 \%$ of children receiving a single agent. In a report of 35 patients sedated for CT by Simpson et al. [9], sedation was done with oral secobarbitol (quinalbarbitone) over a 40-month period. Based on our audit and 3 of the abovementioned studies $[4,7,8]$, midazolam appears to be the most commonly used sedative drug.

Similarly to Mazhar et al. [7], we used oral and IV routes, while Chu et al. [4] used the IM route in addition to oral and IV routes. The route of administration of sedative agents did not influence the quality of MRI images in our audit.

The majority of our sedations were done as planned procedures, similarly to the studies by Mazhar et al. [7] in New York (56 patients, 18-month period) and by Chu et al. [4] in Hong Kong (120 patients, 7-month period). However, unlike these 2 audits, in all our patients a single sedative agent was used, which was mainly midazolam (95.7\% of cases). Only a few patients were sedated with paraldehyde (4.3\%). Mazhar and co-workers [7] used midazolam and chlorhydrate as sedative agents in their audit, with $98 \%$ of the patients receiving $50-100 \mathrm{mg} / \mathrm{kg}$ oral chloral hydrate, while $82 \%$ received $0.1 \mathrm{mg} / \mathrm{kg}$ of IV midazolam. In the audit by Chu et al. [4], $65 \%$ of patients had successful sedations with 
a single sedative agent, $34 \%$ with 2 sedative agents and $1 \%$ with 3 agents. In the study by Simpson and co-workers [9], $91.4 \%$ of patients had adequate sedation with oral secobarbitol as a single agent and failure of sedation in patients younger than 5 years was caused by problems of administration, while in patients older than 5 years it was due to paradoxical excitement.

Malviya et al. [8] did an audit of children who received both sedation and general anaesthesia for both CT and MRI; we evaluated only children who were sedated as this protocol is accepted in our institution. Sedation was unsuccessful in $7 \%$ of children undergoing CT and MRI in the study by Malviya et al. [8]. In contrast, we noted only one instance $(1.4 \%)$ of unsuccessful sedation in which re-scanning was required. A higher success rate in our audit could be due to failure of administration or error in judgement on the side of the practitioner in the audit by Malviya et al. [8].

The quality of images acquired was generally good (88.5\%) with no or minimal motion blur, (Figure 3) and only 1 patient required a repeat examination of the abdominopelvic region due to motion blur (Figure 6). Our percentage of successful sedations was higher $(88.5 \%$ versus $84 \%)$ and our number of patients requiring repeat scan was lower (1 versus 4) in comparison to the study by Mazhar et al. [7]. The number of repeat scans was higher in the audit by Malviya et al. [8], where 7 patients (15\%) had a repeat scan due to motion blur in the images after sedation.

All sedations were administered by the paediatric anaesthesiologist in this audit. Sury et al. [10], in the UK, reported a failure rate of $5 \%$ (61 patients) but no adverse effects relating to airway or breathing complications in a 30-month audit of 1,155 children who were sedated by nurses using oral chlorhydrate, temazepam and droperidol as instructed by the radiologist. They concluded that it was possible to have a nurse lead the sedation service for MRI of children, which was both successful and safe.
In Nigeria, sedations are usually administered by anaesthesiologists and radiologists, with only few sedations carried out by nurses and other trained medical personnel [3]. The higher success rate in our audit, compared to that by Sury et al. [10], could be attributed to the difference in personnel who administered sedation.

In this study, the median duration of MRI examinations was 29.5 minutes with a range of 20 minutes to $70 \mathrm{~min}$ utes. The maximum total scanning time for the MRI procedure did not exceed 50 minutes in the audit by Chu et al. [4]. The difference in scan time can be due to the difference in magnetic field strengths of MRI devices used in the two institutions, as it has been shown that the higher the magnetic strength of the MRI scanner, the faster is the scan time. However, Chu et al. [4] did not reveal the magnetic strength of their MRI machine. All the patients completed their scans after sedation.

Monitoring during the scanning was quite challenging because of the non-availability of MRI compatible monitors. A limitation of this audit is the fact that we could not get a record of the number of additional doses of midazolam required and therefore interruptions made during the MRI procedure. This would have given us an idea on the adequacy of the initial loading dose of our sedatives.

\section{Conclusions}

In conclusion, midazolam (IV or oral) is an adequate sedative and can be used as a single agent for paediatric patients requiring sedation during MRI. Therefore, it is the drug of choice in our institution. No record of adverse events in our 5-year audit was noted and sedation was usually planned and administered by the anaesthesiologist. However, a comparative study of the various drugs used for paediatric MRI sedations is recommended to provide information on the strength of each sedation drug and their relative strengths.

\section{References:}

1. Ogbole GI, Adeleye AO, Adeyinka AO, Ogunseyinde OA: Magnetic resonance imaging: Clinical experience with an open low field strength scanner in a resource challenged African state. J Neurosci Rural Pract, 2012; 3(2): 137-43

2. Del Pizzo B: Advantages and disadvantages of paediatric sedation in magnetic resonance imaging. Radiol Tech, 2016; 84(3): 329-82

3. Omisore $\mathrm{AD}$, Adenekan AT, Ayoola $\mathrm{OO}$ et al: Sedation practice among Nigerian Radiology residents. Southern African Journal of Anaesthesia and Analgesia, 2016; 1(1): 1-6

4. Chu WCW, Lam WWM: Sedation of paediatric patients undergoing magnetic resonance imaging examination: A clinical audit. J HK Coll Radiol, 2002; 5: 176-82

5. De Jonghe B, Cook D, Appere-de-Vecchi C et al: Using and understanding sedation scoring systems: a systematic review. Intensive Care Med, 199; 27: 1325-29
6. Aldrete JA: The post anaesthesia recovery score revisited. J Clin Anaesth, 1995; 7(1): 89-91

7. Mazhar H, Amin S, Heaton P: Audit of imaging under sedation in children. Arch Dis Child, 2012; 97: Al13

8. Malviya S, Voepel-Lewis T, Eildevik OP et al: Sedation and general anaesthesia in children undergoing MRI and CT: Adverse events and outcomes. Br J Anaesth, 2000; 84(6): 743-48

9. Simpson JH, West CD, Law PJ: Paediatric sedation for CT scanning: the safety and efficacy of quinabarbitone in district general hospital setting. Br J Radiol, 2000; 73(665): 7-9

10. Sury MRJ, Hatch DJ, Deeley T et al: Development of a Nurse led sedation service for paediatric magnetic resonance imaging. Lancet, 1999; 353: 1667-71 